\title{
Uso de Técnicas de Inteligência Artificial num Sistema de Mesa Tangível
}

\author{
Evandro Preuss ${ }^{1}$, Dante Augusto Couto Barone ${ }^{1}$, \\ Renato Ventura Bayan Henriques ${ }^{1}$ \\ ${ }^{1}$ Programa de Pós-Graduação em Informática na Educação \\ Universidade Federal do Rio Grande do Sul (UFRGS) \\ Porto Alegre - RS - Brasil \\ evandro.preussegmail.com, baronedinf.ufrgs.br, \\ renatobayanegmail.com
}

\begin{abstract}
The use of technological resources in the teaching-learning processes is an increasingly present reality, including with systems that use artificial intelligence (AI) techniques. It provides learning ways adapted to the student's individual needs. The use of AI in educational systems must consider the norms and the ethical aspects of the responsible use of this type of technology. This article presents a description of the incorporation of AI techniques in a platform based on a tangible tabletop used in inclusive education, considering the ethical implications of incorporating AI into educational systems.
\end{abstract}

Resumo. A utilização de recursos tecnológicos nos processos de ensinoaprendizagem é uma realidade cada vez mais presente, inclusive com sistemas que utilizam técnicas de inteligência artificial (IA). Eles possibilitam trilhas de aprendizagem adaptadas às necessidades individuais dos alunos. O uso de IA em sistemas de ensino deve levar em conta as normas e os aspectos éticos do uso responsável deste tipo de tecnologia. Este artigo apresenta um relato da incorporação de técnicas de IA numa plataforma baseada numa mesa de interação tangivel utilizada na educação inclusiva, considerando as implicações éticas da incorporação de IA em sistemas de ensino.

\section{Introdução}

Nos últimos anos, uma série de avanços tecnológicos foram desenvolvidos e incorporados no processo de ensino-aprendizagem nas escolas, porém o uso da inteligência artificial (IA) nos sistemas que apoiam a educação ainda é modesto. Existem diversas iniciativas para o uso dessa tecnologia para oferecer tutores virtuais, ensino adaptativo, personalização do ensino e interpretação dos dados para avaliar o desempenho e detectar possíveis problemas no aprendizado.

O uso de IA nos processos educacionais geram complexos dilemas e inúmeras questões éticas que não podem ser ignoradas ao se decidir pelo seu uso. Um aspecto importante é que esses sistemas são programados e desenvolvidos por humanos e utilizam alguma técnica de IA que tenta reproduzir o raciocínio humano para uma tomada de decisão. Para isso, é necessário que o sistema seja alimentado por uma quantidade significativa de dados para ser capaz de propor ou tomar novas decisões, o 
que pode gerar um viés, tendência ou discriminação, tanto pela técnica utilizada quanto pelos dados fornecidos.

Uma questão relevante a ser considerada é se estamos preparados para deixar o sistema tomar a decisão sobre questões importantes, especialmente no ensino, como identificar se um aluno realmente aprendeu um conteúdo ou não, detectar se ele precisa de uma aula ou um material de reforço ou até mesmo avaliar se ele pode ser aprovado ou não. Se isso já é um assunto complicado para um professor, será que os sistemas computacionais já têm essa capacidade? Na educação, essas questões éticas envolvendo IA estão presentes e precisam ser identificadas e abordadas.

Este artigo apresenta um relato da utilização de técnicas de IA num sistema já existente, baseado em uma mesa de interação tangível utilizada em processos de educação inclusiva, especialmente para crianças com deficiência intelectual ou autismo. Neste contexto, são analisadas as opções do uso de IA na educação e os aspectos éticos da incorporação dessas técnicas em sistemas que auxiliam o processo de aprendizagem.

\section{Sistemas autônomos e inteligentes}

Inteligência artificial (IA) é uma área da ciência da computação que se propõe a pesquisar e desenvolver dispositivos que simulem a capacidade humana de raciocinar, perceber, resolver problemas e tomar decisões de forma autônoma. Entre várias outras manifestações possíveis, estão a capacidade de apresentar dilemas, propor ou resolver problemas que ainda são exclusivos da inteligência humana (Russell, 2013).

O termo "inteligente" pode ser utilizado para sistemas computacionais que usam algoritmos e dados para tratar problemas e situações complexas, inclusive com a capacidade de melhorar seu desempenho com base na avaliação de decisões anteriores. Estes sistemas podem ser considerados "autônomos" se eles forem capazes de realizar suas tarefas mesmo com mudanças no ambiente de atuação (Karachalios, 2019).

O MIT Media Lab apresentou o conceito de "inteligência ampliada", na qual os seres humanos usam máquinas para facilitar a resolução de problemas práticos, como reconhecimento de padrões, aceleração e otimização da tomada de decisão ou criação de veículos autônomos. Esse trabalho conjunto de humanos e máquinas pode ser considerado uma manifestação de uma forma simples de "inteligência estendida". De acordo com a estrutura conceitual proposta pelo MIT Media Lab:

\footnotetext{
Ao invés de pensar sobre inteligência de máquina em termos de seres humanos versus máquinas, devemos considerar o sistema que integra seres humanos e máquinas - não inteligência artificial, mas inteligência estendida. Ao invés de tentar controlar, projetar ou até mesmo entender sistemas, é mais importante projetar sistemas que participem como elementos responsáveis, conscientes e robustos de sistemas ainda mais complexos. E devemos questionar e adaptar nossos propósitos e sensibilidades como projetistas e componentes do sistema para uma abordagem muito mais humilde: humildade sobre o controle (Karachalios, 2019, p. 3, tradução nossa).
}

\subsection{Sistemas inteligentes na educação}

A aplicação da inteligência artificial na educação tem sido objeto de pesquisas acadêmicas há mais de trinta anos, envolvendo as ciências da aprendizagem (educação, psicologia, neurociência, linguística, sociologia e antropologia) para promover o 
desenvolvimento de ambientes adaptativos de aprendizagem e outras ferramentas flexíveis, inclusivas, personalizadas, envolventes e eficazes (Luckin, 2019).

As aplicações das técnicas de IA na educação fornecem estruturas para otimizar o papel de mediador do professor, auxiliando-o na sua prática didática com a utilização dos computadores como uma forma de inteligência estendida, garantindo um aprendizado mais completo e significativo para os alunos. A aplicação de sistemas inteligentes na educação pode trazer inúmeros benefícios em diferentes etapas e abordagens, tais como:

a) Plataformas adaptativas: as ferramentas de ensino são capazes de propor trilhas de aprendizado individualizadas, com metodologias e técnicas que analisam o comportamento do aluno e permitem avançar o conteúdo de acordo com regras pré-estabelecidas. A plataforma também registra todas as informações de cada experiência de uso e propõe um caminho específico para cada aluno a partir do seu ponto de partida e da análise da base de dados dos demais alunos, estabelecendo relações entre as evoluções dos usuários de acordo com a análise de interações anteriores.

b) Supervisão da aprendizagem: a IA também pode ser utilizada pelos professores para avaliar o desempenho e monitorar a aprendizagem de cada aluno de forma individualizada, através de métodos estatísticos que detectam problemas e permitem atuar de forma rápida e eficiente.

c) Educação personalizada: cada estudante apresenta características individuais que precisam ser respeitadas. $\mathrm{O}$ uso de sistemas tutores inteligentes permite traçar um perfil com as características dos alunos e dar aos estudantes a autonomia para conduzir os estudos.

d) Gestão pedagógica: como as técnicas de IA são capazes de coletar, cruzar e interpretar um grande volume de dados, elas podem analisar o desempenho dos estudantes em avaliações internas e externas, identificando os conteúdos que eles realmente dominam, bem como eventuais lacunas em sua aprendizagem.

A IA envolve programas de computador desenvolvidos para interagir com o mundo de maneira que normalmente exige raciocínio e inteligência humana. A IA depende tanto do conhecimento sobre o mundo quanto dos algoritmos para processar inteligentemente esse conhecimento. Esse conhecimento sobre o mundo é representado nos chamados "modelos". Existem três modelos que envolvem a IA na educação (Luckin, 2019):

a) Modelo pedagógico: envolve as abordagens efetivas para o ensino, com o conhecimento e experiência de ensinar;

b) Modelo de domínio: envolve o assunto sendo aprendido e o conhecimento do mesmo;

c) Modelo de aprendiz: envolve o aluno e o conhecimento que ele já tem.

As interações representadas no modelo, como as atividades atuais do aluno, avanços anteriores, estado emocional e feedback podem ser usadas pelos componentes de domínio e pedagogia para inferir o sucesso do aprendiz. Os modelos de domínio e pedagogia também usam essas informações para determinar a próxima interação mais 
apropriada, pois as atividades do aluno são continuamente inseridas no modelo do aprendiz, tornando o modelo mais rico e mais completo e o sistema "mais inteligente" (Luckin, 2019).

Alguns sistemas apresentam para os alunos e professores os resultados da análise com informações valiosas sobre as realizações e progressos do aluno e eventuais equívocos que eles tenham cometido. Isso auxilia os professores a entenderem a abordagem dos alunos ao aprendizado e permite que eles moldem adequadamente as futuras experiências de ensino. Para os alunos, estes modelos permitem acompanhar o seu próprio progresso e podem ajudar na motivação, incentivando-os a refletir sobre suas aprendizagens.

Uma das vantagens dos sistemas de IA adaptativos é que eles normalmente coletam uma grande quantidade de dados que podem ser computados para melhorar dinamicamente a pedagogia e os modelos de domínio. Esse processo ajuda a encontrar novas maneiras de fornecer suporte mais eficiente, personalizado e contextualizado, enquanto também testa e aprimora o entendimento dos processos de ensino e aprendizagem.

\section{Aspectos éticos do uso de IA}

O desenvolvimento e aplicações da IA está se acelerando em todas as áreas e permeando todos os aspectos de nossas vidas. Um dos problemas éticos enfrentados no uso de IA está relacionado às questões de privacidade, uma vez que o compartilhamento de dados é essencial para a integração de sistemas. Não está esclarecido, ainda, quais são as implicações dos métodos, tecnologias e ideologias que sustentam a geração, análise, interpretação e uso de dados dos sistemas educacionais que utilizam IA.

Os algoritmos que sustentam os sistemas de IA precisam ser transparentes, interpretáveis e previsíveis e precisam também ser capazes de explicar seu comportamento de modo que os seres humanos possam entender, desde a forma como eles interpretaram os dados até o porquê eles recomendaram uma saída específica (Rossi, 2016).

Os princípios gerais que envolvem os sistemas autônomos e inteligentes (Autonomous and Intelligent Systems - A/IS) são (IEEE, 2019):

a) Direitos humanos: os A/IS devem ser criados e operados para respeitar, promover e proteger os direitos humanos reconhecidos internacionalmente.

b) Bem-estar: os desenvolvedores de A/IS devem promover o bem-estar humano como primeiro critério no sistema.

c) Agência de dados: os criadores de A/IS devem capacitar os indivíduos para acessar e compartilhar com segurança seus dados, garantindo o controle sobre os dados das pessoas e a privacidade.

d) Eficácia: os criadores e operadores de A/IS devem fornecer evidência da eficácia e adequação à finalidade do uso de IA.

e) Transparência: a base para uma determinada decisão do A/IS deve ser sempre detectável e verificável. 
f) Prestação de contas: A/IS devem ser criados e operados para fornecer uma justificativa inequívoca para todas as decisões tomadas.

g) Conscientização sobre o uso indevido: os criadores do A/IS devem proteger-se contra todos os potenciais abusos e riscos do uso e operação do mesmo.

h) Competência: os criadores do A/IS devem especificar e os operadores devem aderir ao conhecimento e habilidade necessários para uma operação segura e eficaz.

A sociedade ainda não estabeleceu padrões universais ou princípios orientadores para incorporar valores em A/IS pois existem complexidades conceituais para definir o que são "valores". Há uma dificuldade para definir valores sociais ou culturais, como "segurança", "autonomia" ou "justiça". As normas, por outro lado, podem ser expressas computacionalmente, uma vez que são observáveis no comportamento humano e podem ser representadas como instruções para agir de formas definidas em contextos definidos e expressas em termos de obrigações e proibições.

Os critérios éticos envolvidos em A/IS são (IEEE, 2019):

a) Para desenvolver um A/IS capaz de seguir normas sociais e morais, o primeiro passo é identificar as normas da comunidade específica na qual os A/IS devem ser implantados e as normas relevantes para os tipos de tarefas e funções dos sistemas.

b) Para responder às mudanças dinâmicas de normas na sociedade, os A/IS ou seus projetistas devem ser capazes de alterar suas normas ou adicionar novas, sendo transparentes sobre essas mudanças para usuários, designers, representantes da comunidade em geral e outras partes interessadas.

c) Os desenvolvedores de A/IS devem identificar as maneiras pelas quais as pessoas resolvem conflitos de normas e as maneiras pelas quais eles esperam que o A/IS resolva conflitos de normas semelhantes.

d) Os A/IS, especialmente aqueles com normas incorporadas, devem ter um alto nível de transparência, mostrando a rastreabilidade no processo de implementação, verificabilidade matemática de seu raciocínio, honestidade em sinais baseados em aparência e inteligibilidade da operação e das decisões dos sistemas.

e) Como os projetistas e desenvolvedores não podem antecipar todas as possíveis condições operacionais e possíveis falhas de A/IS, várias estratégias para mitigar a chance e a magnitude do dano devem estar implementadas;

f) O processo de identificação da norma deve documentar as semelhanças e diferenças entre as normas que os seres humanos aplicam a outros seres humanos e as normas que eles aplicam aos A/IS.

\section{Uso de IA num sistema de interação tangível}

As interfaces tangíveis (Ishii, 2008) têm sido utilizadas para interação humanocomputador (Marco, 2009; Marshall, 2007), especialmente no processo educacional inclusivo, para atender crianças com deficiência intelectual ou autismo. As interfaces 
tangíveis podem ser definidas como qualquer interface onde o usuário interfere no sistema digital através de dispositivos físicos ou objetos reais (Ishii, 2008). É uma interface na qual o ambiente e o usuário são utilizados como meio de interação, utilizando objetos físicos para interagir com sistemas digitais e transformando uma ação física em uma ação virtual.

Uma mesa tangível (fig. 1) é uma interface que permite interações em sua superfície utilizando objetos físicos reais, identificados na sua base com marcadores fiduciais para reconhecimento das interações. Ela pode ser utilizada em tarefas cognitivas que envolvem representações visuais, sonoras, táteis e simbólicas de objetos. Uma das vantagens da utilização de interfaces interativas tangíveis é seu manuseio fácil e intuitivo e a possibilidade de inserir, numa mesma interface, múltiplas formas de interação e de representação sonora, visual ou tátil, o que permite atender a diversidade humana em termos de deficiência.

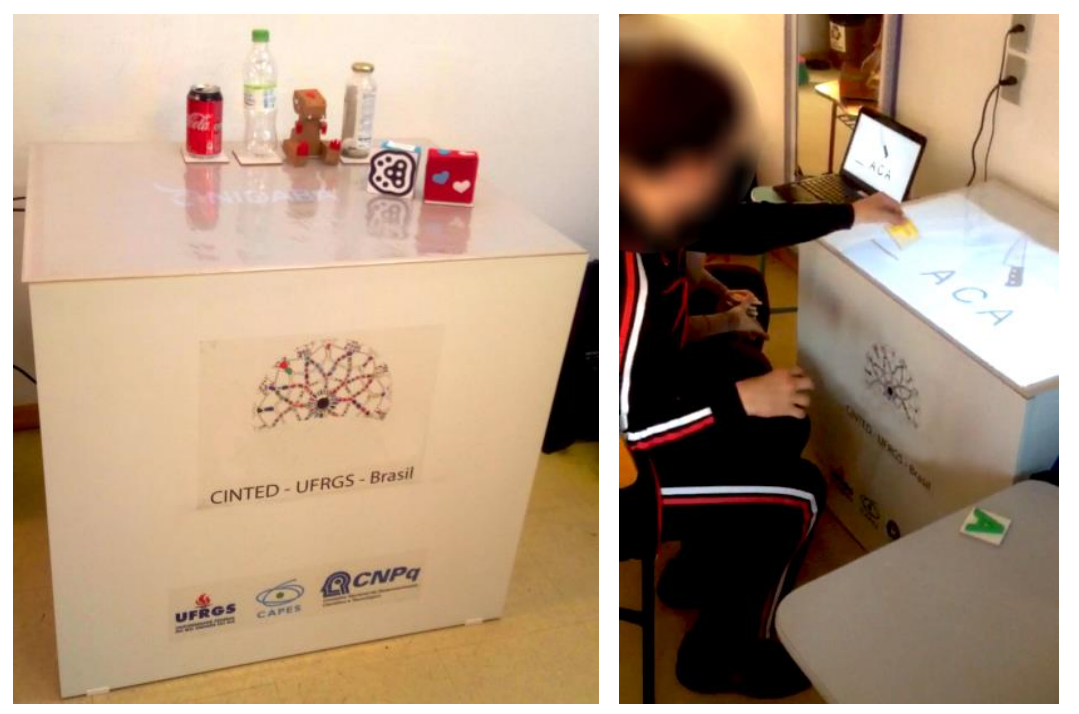

Figura 1. Mesa tangível com os objetos e marcadores fiduciais e seu uso

Um dos problemas encontrados no uso da mesa tangível é que as atividades criadas para ela apresentam os mesmos níveis de dificuldade, facilidades e assistência para todos os tipos de alunos. Devido à grande diferença de capacidade e de déficits, especialmente das crianças com transtorno do espectro autista ou deficiência intelectual, é necessária a criação de diferentes atividades para os diferentes alunos.

Neste contexto, este trabalho relata o processo de incorporação das técnicas de IA no ambiente tangível já desenvolvido (GLUZ et al, 2018; PREUSS et al, 2019) para transformá-lo numa plataforma adaptativa, propondo diferentes trilhas de aprendizado individualizadas. Essa inclusão de técnicas proporciona uma educação personalizada e facilita a supervisão da aprendizagem e a gestão pedagógica, considerando as implicações éticas do uso de IA em sistemas, especialmente por se tratar de uma ferramenta assistiva para uso na educação inclusiva.

O sistema desenvolvido faz parte de uma pesquisa-ação e foi desenvolvido baseado em métodos ágeis, envolvendo processo incremental, com a criação de protótipos funcionais do sistema. Durante esse processo, nos últimos dois anos, a cada nova versão, o sistema e o editor eram utilizados e suas funcionalidades testadas e 
aprimoradas. A usabilidade do editor e a autonomia e motivação para uso da plataforma foram avaliados através dos questionários SUS (System Usability Scale) e IMI (Intrinsic Motivation Inventory). A aceitação e uso desta plataforma pelos professores ainda está sendo avaliada com os questionários TAM (Technology Acceptance Model) e TPACK (Technological Pedagogical Content Knowledge).

Neste sistema, as aplicações para mesa tangível são criadas através de um editor especificamente desenvolvido para esse fim. Elas são formadas por um conjunto de cenas, nas quais são escolhidas as imagens de fundo, os áudios de narração ou explicação e as demais imagens e animações que compõem a aplicação. Quando há alguma interação com os objetos tangíveis sobre a mesa, são delimitadas as áreas dessa interação, a configuração dos elementos de marcação fiduciais corretos e incorretos e o respectivo feedback sonoro ou pictográfico para cada resposta. De acordo com as respostas, uma nova cena pode ser exibida. Originalmente, uma atividade desenvolvida apresentaria sempre o mesmo comportamento e feedback, independentemente do tempo de uso ou da quantidade de acertos ou erros que o aluno cometesse.

A figura 2a apresenta a interface do editor de aplicações para a mesa tangível Eduba (PREUSS et al, 2019) com as áreas de interação (quadrados com bordas vermelhas) onde devem ser colocadas os respectivos objetos sobre a mesa, sua definição dos marcadores fiduciais corretos e incorretos e os respectivos áudios e imagens de feedback. Dependendo do objeto colocado sobre essas áreas, um feedback pictográfico (no quadrado com borda azul) ou sonoro é apresentado. Para cada cena, suas propriedades podem ser definidas e configuradas (fig $2 b$ ).

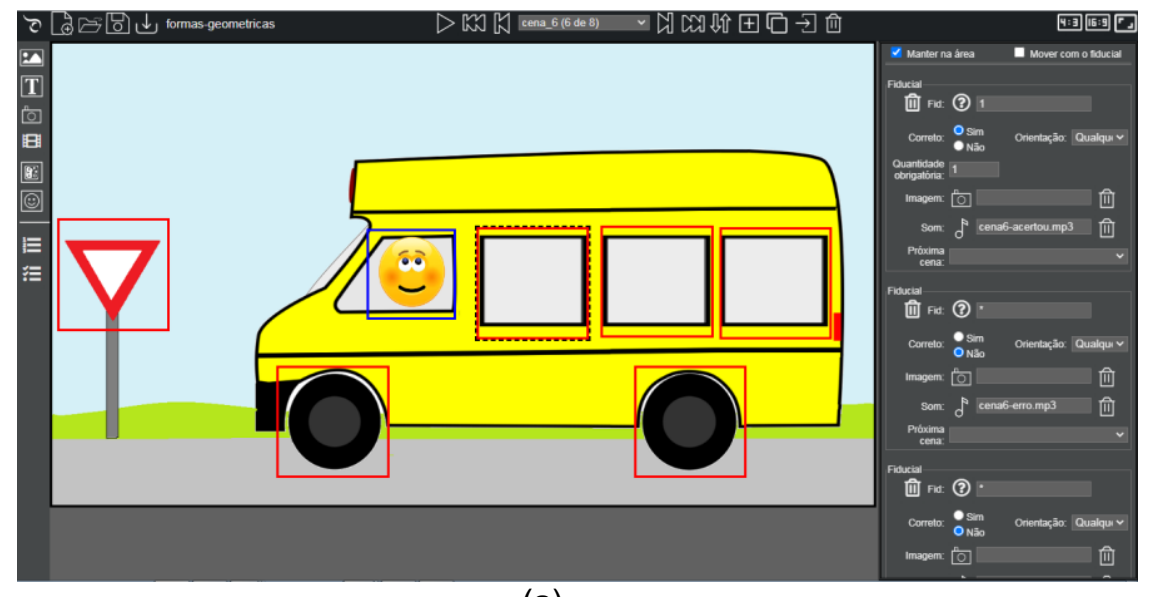

(a)

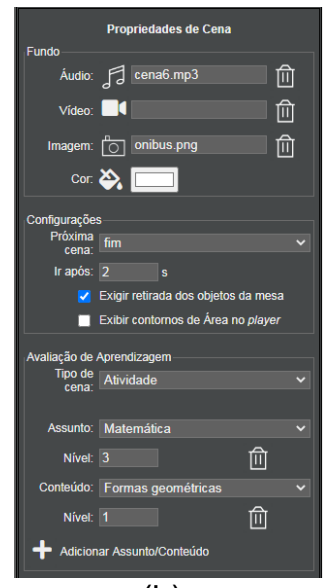

(b)

Figura 2. Interface do editor com as áreas de interação e propriedades

Para incorporar técnicas de IA foram realizadas alterações no editor de aplicações para permitir criar, além das cenas de atividades, as cenas de lições e dicas e especificar o tema, conteúdo, assunto e nível de dificuldade (fig 2b). Diferentes ações também podem ser definidas de acordo com a quantidade de erros cometidos em cada área de interação. Na plataforma de ensino e execução da aplicação, foi permitido cadastrar e identificar os alunos e definir quais atividades cada um deve realizar. Também foram criados agentes para a realização do registro e análise de todas as informações de uso, interações nas áreas das cenas, dos dados de acertos, erros e evoluções da aprendizagem, de forma individualizada. 
O agente criado pode repetir as instruções sonoras, exibir as lições de reforço ou as dicas adicionais quando for detectado que o aluno está com dificuldade na resolução, demorando para responder, apresentando erros constantes ou quando ele explicitamente solicitar a ajuda. O sistema pode também avançar automaticamente para lições mais avançadas quando detectar que o aluno está com um alto índice de acertos. Isso é importante, especialmente para o uso num contexto inclusivo, pois cada aluno tem o seu desempenho avaliado e comparado com o próprio desempenho em outras atividades e em usos anteriores, de acordo com o assunto, tema, conteúdo e nível de dificuldade. Isso garante a eficácia com respeito às diferenças, aos direitos humanos e ao bem-estar do aluno.

Todas as interações no sistema são registradas e formam uma base de conhecimento, que é usado pelo agente para determinar os tempos e ações das próximas interações. Além disso, ao final de cada atividade ou tarefa, o sistema permite registrar uma avaliação pelo professor ou mediador da atividade, informando se, na avaliação dele, o aluno cumpriu ou não (no todo ou em parte) com o objetivo da atividade. Esta informação também é incorporada na base de dados e é considerada pelo sistema na avaliação da tomada de decisão para propor diferentes ações para o aprendizado.

O sistema pode sugerir repetições na execução das atividades quando o desempenho não é adequado ou pode ser melhorado. Ele também registra e alerta quando o aluno não está realizando a tarefa com a devida atenção, não aguardando o término das instruções e orientações da atividade ou realizando as interações baseadas em tentativa e erro. O sistema também monitora a ocorrência de eventuais falhas ou exceções e informa ao operador em caso de eventuais anomalias.

Para garantir a transparência e a prestação de contas sobre as decisões tomadas, o sistema mantém um registro e pode fornecer relatórios de todas as tomadas de decisão, juntamente com os dados e regras que foram utilizadas para as mesmas. O sistema registra e compara a avaliação do professor com as análises do sistema, como um mecanismo de proteção, para evitar que sejam incorporadas regras que possam gerar um viés, tendência ou discriminação, especialmente porque a base de dados não é grande o suficiente para garantir que exceções não se tornem a regra.

Para garantir os critérios de conscientização sobre o uso indevido, o sistema exige que somente pessoas habilitadas e qualificadas possam utilizar os recursos e alimentar o sistema, com proteções de identificação dos usuários e uso de senhas. Para garantir os aspectos de competência do uso do sistema, o mesmo oferece os manuais de uso, sistemas de ajuda, tutoriais e material para formação e treinamentos para professores e mediadores que utilizarão a plataforma adaptativa de interação tangível.

\subsection{Resultados}

A incorporação de técnicas de IA para transformar o sistema de mesa tangível numa plataforma adaptativa foi uma necessidade detectada no início da pesquisa. As técnicas de IA foram incorporadas no sistema considerando as implicações éticas do uso deste tipo de tecnologia e respeitando as individualidades de cada estudante. Foi criado um agente, transformando o sistema numa plataforma adaptativa, para permitir a supervisão da aprendizagem, educação personalizada e gestão pedagógica. Com isso, o sistema é capaz de propor diferentes trilhas de aprendizado na execução das atividades na mesa 
tangível, além de fornecer relatórios de desempenho individualizado nas atividades de acordo com o assunto, tema, conteúdo e nível de dificuldade.

Um dos problemas enfrentados ao incorporar essas características no sistema é que aumentou a complexidade na criação das aplicações, necessitando criar e definir as cenas das lições de reforço e dicas, além de especificar o tema, assunto e nível. Para garantir a facilidade de criação originalmente proposta pela plataforma, o sistema ainda permite a construção de aplicações sem esse recurso. Se elas estão disponíveis, o sistema sugere e atua, caso contrário, ele mantém o roteiro sequencial da atividade proposta pelo professor.

Algumas aplicações foram desenvolvidas e o sistema sem as técnicas de IA já está sendo usado e avaliado há dois anos. As avaliações já realizadas demonstram a utilidade e viabilidade do uso de interação tangível na educação inclusiva e a necessidade de uma plataforma adaptativa.

Duas mesas tangíveis foram desenvolvidas e instaladas na sala de recursos do atendimento educacional especializado em duas escolas públicas da rede municipal de ensino. Dezesseis professores destas escolas estão participando de uma formação para uso desta tecnologia. Eles ainda estão criando as atividades para uso na educação inclusiva e participam da pesquisa sobre a usabilidade e aceitação do uso do sistema.

Os alunos já utilizaram o sistema de interação tangível e as aplicações por nós desenvolvidas para testar o uso da mesa e a viabilidade da nossa proposta. A validação, os eventuais ajustes necessários e os resultados finais do uso desta plataforma adaptativa na educação inclusiva ainda estão sendo realizadas e necessitam pelo menos de 12 a 18 meses.

\section{Conclusão}

O crescente uso dos recursos tecnológicos no processo de ensino-aprendizagem, especialmente na educação inclusiva, é uma realidade e uma necessidade. A incorporação do uso de técnicas de IA nos sistemas pode trazer consideráveis vantagens, como a personalização e adaptação do ensino para cada aluno, de acordo com seus conhecimentos e capacidades.

Este artigo apresentou os aspectos relacionados à incorporação de técnicas de IA num sistema de apoio ao processo de ensino-aprendizagem no contexto inclusivo baseado numa mesa de interação tangível. Isto permitiu aprimorar o sistema desenvolvido para criação e uso de aplicações para mesa tangível, transformando-o numa plataforma adaptativa que proporciona uma educação personalizada e é capaz de propor diferentes trilhas de aprendizado, além de facilitar a supervisão da aprendizagem e gestão pedagógica.

A incorporação de técnicas de IA no sistema considerou as possibilidades e implicações de uso de IA na educação e os aspectos éticos que devem ser respeitados ao incorporar estas técnicas no sistema. A técnica utilizada respeita as individualidades e características de cada aluno para avaliar a aprendizagem pois só analisa e compara o seu desempenho com os próprios resultados em atividades anteriores, de acordo com o tema, assunto e nível de dificuldade. 
Os resultados parciais demonstram a utilidade e viabilidade do sistema de mesa tangível como uma plataforma adaptativa, especialmente na educação inclusiva, com alunos com deficiência intelectual ou autismo. A pesquisa e coleta de dados ainda se estenderá nos próximos dois anos para aperfeiçoar os algoritmos utilizados, as regras definidas e o próprio sistema.

\section{Agradecimentos}

O presente trabalho foi realizado com apoio do Ministério da Ciência, Tecnologia e Inovações, da Coordenação de Aperfeiçoamento de Pessoal de Nível Superior - Brasil CAPES e do Conselho Nacional de Desenvolvimento Científico e Tecnológico CNPq.

\section{Referências}

Gluz, J. et al. (2018) “Ambiente Virtual Tangível para Integração Sensorial no Ensino de Ciências numa Perspectiva Inclusiva", Simpósio Brasileiro de Informática na Educação - SBIE, v. 29, n. 1, p. 545. DOI: 10.5753/cbie.sbie.2018.545.

IEEE (2019) "Ethically aligned design: a vision for prioritizing human well-being with autonomous and intelligent systems". IEEE. Disponível em: https://engagestandards. ieee.org/rs/211-FYL-955/images/EAD1e.pdf. Acesso em: 03 jul. 2020.

Ishii, H. (2008) “The Tangible User Interface and its Evolution”. ACM 51, 6, p. 32-36.

Karachalios, K. and Ito, J. (2019) "Human Intelligence and Autonomy in the Era of 'Extended Intelligence'". Council on Extended Inteligence. Disponível em: https://globalcxi.org/wp-content/uploads/CXI_Essay.pdf. Acesso em: 20 jun. 2020.

Luckin, R. et al. (2019) "Intelligence Unleashed: an argument for AI in Education". Disponível em: https://www.pearson.com/content/dam/corporate/global/pearson-dotcom/files/innovation/Intelligence-Unleashed-Publication.pdf. Acesso em 21 jun. 2020 .

Marco, J. et al. (2009) "Bringing Tabletop Technologies to Kindergarten Children". In: BCS Conference on Human Computer Interaction, 23, Cambridge University. Proceedings. p. 103-111.

Marshall, P. (2007) “Do tangible interfaces enhance learning?” In: International Conference on Tangible and Embedded Interaction, ACM. New York. Proceedings. p. $163-170$.

Preuss, E. et al. (2019) "E-DUB-A: A Tangible Educational Resource Editor in Inclusive Classes". 2019 IEEE 19th International Conference on Advanced Learning Technologies (ICALT), Maceió, Brazil, 2019, pp. 303-307, DOI: 10.1109/ICALT.2019.00095.

Rossi, F. (2016) “Artificial Intelligence: Potential Benefits and Ethical Considerations”. Policy Department C: Citizens' Rights and Constitutional Affairs, European Union. Disponível em: http://www.europarl.europa.eu/RegData/etudes/BRIE/2016/571380/ IPOL_BRI(2016)571380_EN.pdf. Acesso em: 26 jun. 2020.

Russell, S. J. and Norvig, P. (2013) "Inteligência artificial". Traduzido por Regina Célia Simille. $3^{\mathrm{a}}$ ed. Rio de Janeiro: Elsevier. 